\title{
Assessment of Pain Perception in Paediatric Patients on Application of Cooled and Uncooled Topical Anaesthetic Gel before Infiltration Anaesthesia - A Pilot Study
}

\author{
Prathyusha P. ${ }^{1}$, Amith Adyanthaya ${ }^{2}$, Marium Raheema ${ }^{3}$, Swetha S. Nair ${ }^{4}$, \\ Aparna Sivaraman ${ }^{5}$, Risana $K^{6}$, Nazreen Ayub K ${ }^{7}$, Reshma Aloysius ${ }^{8}$
}

1, 2, 3, 4, 5, 6, 7, 8 Department of Paediatric and Preventive Dentistry, KMCT Dental College, Mukkam, Calicut, Kerala, India.

\section{ABSTRACT}

\section{BACKGROUND}

Topical anaesthesia is fundamental in enhancing pain control during the process of dental injections. The study compared the effect of cooled and uncooled topical anaesthetic gel before infiltration anaesthesia in assessing pain perception in paediatric patients undergoing dental treatment procedures.

\section{METHODS}

This is a split-mouth study where 16 children aged between 8 and 10 years who required bilateral local anaesthesia administration for various dental procedures were selected. Before infiltration anaesthesia, topical anaesthetic gel cooled to $4^{\circ} \mathrm{C}$ was applied on one side in the first visit followed by application of uncooled gel on the contralateral side in the subsequent visit. The patients were asked to individually rate their pain experience on each side using the Wong-Baker Faces Pain Rating Scale (WB-FPRS). Anxiety was assessed using pulse oximeter and FLACC scale (face, legs, activity, cry, and consolability). Data was analysed statistically using the paired ' $\mathrm{t}$ '-test and a $\mathrm{P}$ value less than or equal to 0.05 was considered as statistically significant.

\section{RESULTS}

Patients reported less pain on Wong-Baker Faces Pain Rating Scale by using cooled topical anaesthetic gel. Discomfort and anxiety levels were also seen to be low when assessed using pulse oximeter and FLACC Scale for the same group $(\mathrm{P}<0.05)$.

\section{CONCLUSIONS}

Cooling the soft tissue site with anaesthetic gel helped reduce pain perception during infiltration in children and was seen to be a more feasible technique. Hence this technique can be used as a successful adjunct to the local anaesthesia administration prior to dental procedures.

\section{KEY WORDS}

Anaesthesia, Cooling, Topical Gel, Injection, Pain

\author{
Corresponding Author: \\ Dr. Swetha S. Nair, \\ Department of Paediatric and \\ Preventive Dentistry, KMCT Dental \\ College, Mukkam, Calicut, \\ Kerala, India. \\ E-mail: swethanr15@gmail.com
}

DOI: $10.14260 / \mathrm{jemds} / 2021 / 362$

How to Cite This Article:

Prathyusha P, Adyanthaya A, Raheema M, et al. Assessment of pain perception in paediatric patients on application of cooled and uncooled topical anaesthetic gel before infiltration anaesthesia - a pilot study. J Evolution Med Dent Sci 2021;10(23):1751-1755, 10.14260/jemds/2021/362

Submission 01-02-2021,

Peer Review 13-04-2021,

Acceptance 20-04-2021,

Published 07-06-2021.

Copyright (c) 2021 Prathyusha P. et al.. This is an open access article distributed under Creative Commons Attribution License [Attribution 4.0 International (CC BY 4.0)] 


\section{BACKGROUND}

Paediatric dentistry emphasizes primarily on local anaesthesia (LA) as the front runner to minimize pain perception during dental procedures. Even with continuous development in new techniques for dental injections, this procedure still causes pain and discomfort and is often described as a major reason for dental anxiety. Studies on topical anaesthesia are quite common in dentistry but with varying results of their outcome. Successful behavior management of the child can be achieved by topical treatment of the tissues to perceive minimum pain during injection of LA. Various methods have been employed to minimize pain perception in children like cooling the topical gel, vibrotactile devices such as Vibraject ${ }^{1}$ and Dental Vibe ${ }^{2}$ that provide mechanical vibrations to the surrounding tissues and act as counter stimulation. These advanced techniques involve high cost, and the complexity of the equipment might further aggravate the child's anxiety and fear.

Application of topical gels does not always guarantee pain free injections, and the numbing effect is dependent on several factors such as speed of injection and gauge size of the needle. ${ }^{3}$ Authors have also stated that anaesthetic gels only relieve pain caused by the needle insertion and not of the actual injection. ${ }^{4}$ Different types of topical gels (e.g. lidocaine, prilocaine or benzocaine) are used before dental procedures in recent times.

Cooling the tissues has a long standing history in medicine. The technique of local external cooling has been in use for treating musculoskeletal pain, fractures, sports injuries, sprains, etc. Studies on topical cooling of oral tissues are limited in dentistry. Although the concept of cooling the soft tissue prior to injection procedures is established, the literature lacks such kind of application in paediatric dental procedures. A study from 1989 reported how the use of ice for cooling on the palate before and during local infiltration anaesthesia relieved discomfort, but no other method of topical anaesthesia was compared to this cooling effect. ${ }^{5}$ Another study revealed that cooling was an effective adjunct to conventional topical anaesthesia but the efficacy of ice as the primary anaesthetic agent was not evaluated. ${ }^{6}$ The mechanism of action for cooling was found to be due to stimulation of myelinated A fibers and in turn activating inhibitory pain pathways. ${ }^{7}$ Furthermore, it was seen to cause decrease in the activation threshold of tissue nociceptors and conduction velocity of nerve signals conveying pain and thereby causing cold-induced neuropraxia.

\section{Objectives}

- To introduce a cost-effective method which is less technique - sensitive but clinically very effective and can replace the traditional methods of inducing local anaesthesia.

- To assess and compare the pain perception on application of cooled and uncooled topical anaesthetic gel during infiltration anaesthesia.

- To assess the pain perception of the patient during dental injections using Wong Bakers Faces Pain Rating Scale in both experimental groups.
- To compare change in Pulse rate and $\mathrm{SpO}_{2}$ levels before and after injections using both cooled and uncooled topical gel.

- To evaluate patients based on FLACC scale after the injection procedure of application of both cooled and uncooled gel.

\section{METHODS}

This is a cross-sectional observational study which was conducted from February to April 2019. The study was conducted in the Department of Paediatric Dentistry after obtaining ethical clearance (IEC / IRB No: KMCTDC / IEC / 2019 / 12). Parents or guardians of the selected subjects were provided with complete details of the study, who willingly allowed their children to participate in the study after signing the consent form. 16 children aged between 8 and 10 years fulfilling the inclusion criteria were selected for the study.

\section{Inclusion Criteria}

1. Patients requiring local anaesthesia for dental extraction of retained maxillary anterior teeth on either side.

2. Cooperative patients (Frankel's behavior rating scale: positive and definitely positive)

3. Healthy patients (ASA 1) who were aged between 8 and 10 years.

\section{Exclusion Criteria}

1. Medically compromised patients

2. Patients with history of allergy to anaesthetic agents

3. Patients with swelling, pain or periapical pathology

4. Patients with uncooperative behaviour.

All patients, who reported to the Department of Paediatric dentistry and were suitable according to the above mentioned criteria were subjected to the procedure at two visits. Local anaesthesia was administered after application of local anaesthetic gel - Stim Lidayn Oraspot gel (benzocaine $20 \%$ ) on the oral mucosa. On the test side of oral mucosa, cooled gel was applied at the first visit prior to the dental injection whereas on the contralateral side anaesthetic gel at room temperature served as the control. All the participating children and their guardians were informed regarding the technique to be performed and consent was obtained. Patients were familiarized with Wong bakers pain rating scale (Figure1), Pulse oximeter (Apex medical systems Hyderabad, India) (Figure 2) and FLACC scale that were used to assess the pain perception after pricking. The tell show do method and communication were used for behavior modification of these children during the procedure. The quadrants were allocated to control and study group according to simple randomization. The soft tissue site was dried with a sterile gauze before application of the anaesthetic gel. The benzocaine $20 \%$ gel was cooled and maintained at $4^{0} \mathrm{C}$. A pepper-corn sized topical anaesthetic gel (Figure 3,4$)$ on $1 \mathrm{~cm}^{2}$ area of the tissue was applied using a cotton pellet for 1 minute and needle was then positioned 
and slowly inserted. The same procedure was followed for uncooled gel, maintained at room temperature. Injection of $1.5 \mathrm{ml}$ of Warren Lignox lignocaine $2 \%$ A with 1:80000 adrenalin using a 24 gauge needle (Dispovan, India) was administered after negative aspiration gradually over $20-30$ seconds for infiltration. Pain or discomfort was graded by the patient on the Wong bakers pain rating scale by pinpointing a particular face that best described their pain perception. The WB-FPRS consists of six drawn figures indicating a range from 'no hurt' to 'hurts worst' (Figure 5). The children were assessed by pulse oximeter (index finger of left hand) and Face Legs Activity Cry Consolability scale prior to and also after the injection procedure.
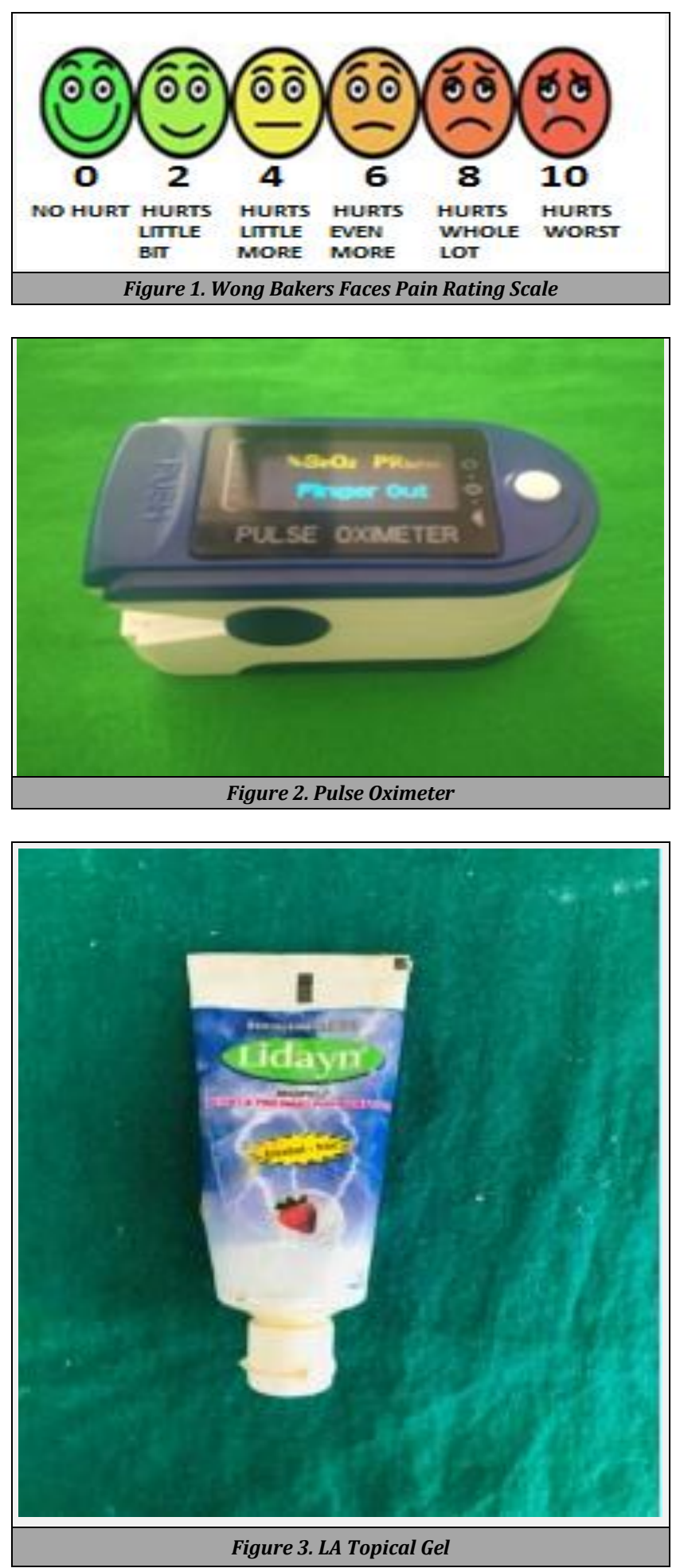

The pulse rate and oxygen saturation levels were recorded 15 minutes before procedure and variations were recorded to calculate the mean value for calibration and assessing range of values. Furthermore readings during application and post infiltration for 1 minute were recorded and mean was calculated. Entire procedure was done by the primary operator and another operator, blinded to the study recorded FLACC scale based on the physical reaction of the subject. After these steps extraction procedure was carried out, which was not evaluated in the study. The first and second appointments were carried out with a minimum of two weeks between them to avoid any persistence of pain.
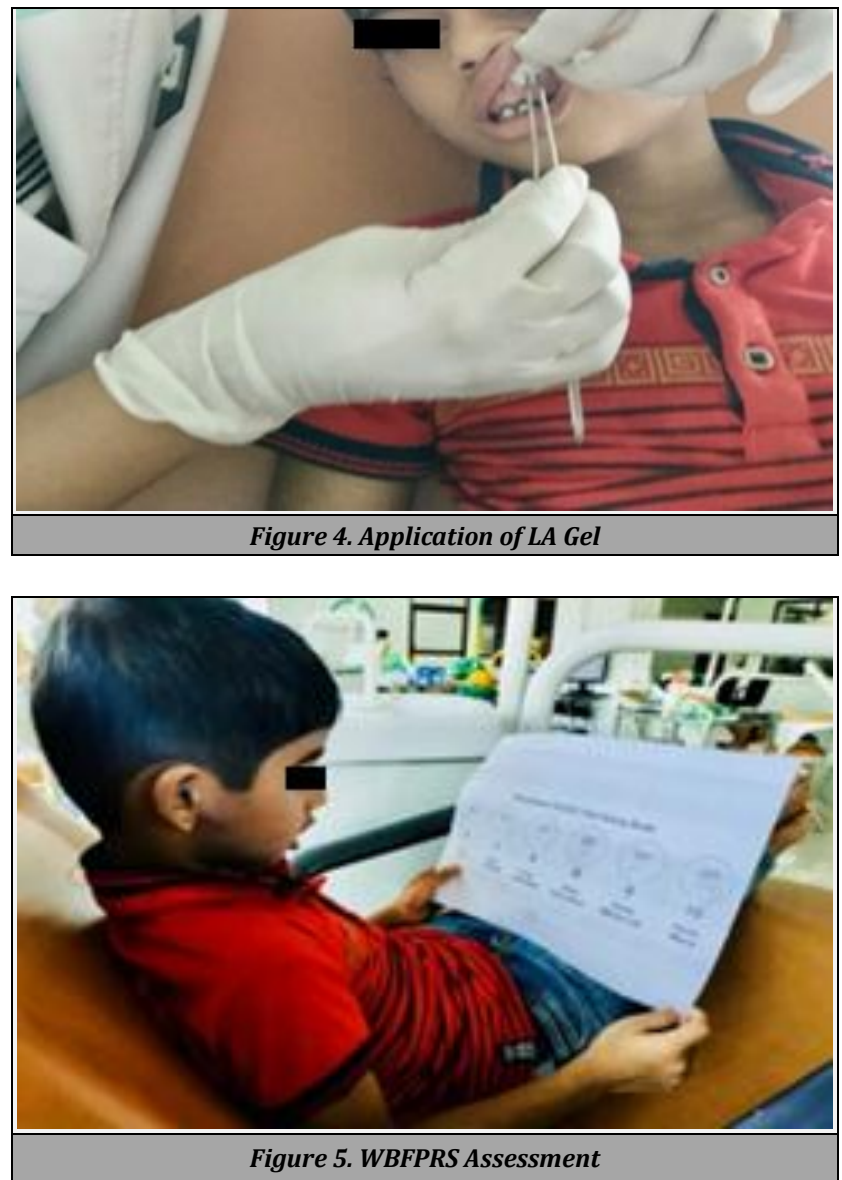

\section{Statistical Analysis}

Analysis of the data was carried out using SPSS version 20.0. Paired ' $t$ ' test was used to compare pain perception during infiltration with cooled or uncooled topical gel. In all the statistical tests used in this study the significant level was pre-set at $\mathrm{P} \leq 0.05$.

\section{RESULTS}

The current research comprised of 16 children aged from 8 to 10 years, who required bilateral local anaesthesia administration for dental procedures. In this pilot study there was a significant difference in the mean values obtained by assessing the reports of the patients on the Wong Bakers Pain Rating Scale. Further evaluation by comparing the pulse rates and $\mathrm{SpO}_{2}$ levels in each child following the dental procedures 
gave a positive difference. The FLACC scale also revealed marked difference in response. Table - 1 represents the comparison between post treatment of uncooled and cooled gel. The differences observed between cooled and uncooled gel were found to be statistically significant $(P<0.05)$. The mean WBPRS, FLACC, PR and PO2 scores for cooled gel were found to be 1.3125, 1.1250, 91.0625 and 95.3125 respectively. And the same for uncooled gel was found to be $2.3125,2.4375,96.0625$ and 97.0 respectively. Statistically significant differences were observed between the two groups, indicating that precooled anaesthetic gel was effective prior to infiltration anaesthesia.

\begin{tabular}{|c|c|c|c|c|c|c|}
\hline \multicolumn{7}{|c|}{ Post-Operative } \\
\hline & Var00001 & $\mathbf{N}$ & Mean & $\begin{array}{c}\text { Std. } \\
\text { Deviation }\end{array}$ & $\begin{array}{c}\text { Std. Error } \\
\text { Mean }\end{array}$ & P Value \\
\hline \multirow{2}{*}{ Wbps } & Cooled gel post & 16 & 1.3125 & .47871 & .11968 & 0.000 \\
\hline & Uncooled gel post & 16 & 2.3125 & .47871 & .11968 & 0.000 \\
\hline \multirow{2}{*}{ Flacc } & Cooled gel post & 16 & 1.1250 & .61914 & .15478 & 0.000 \\
\hline & Uncooled gel post & 16 & 2.4375 & .81394 & .20349 & 0.000 \\
\hline \multirow[b]{2}{*}{$\operatorname{Pr}$} & Cooled gel post & 16 & 91.0625 & 3.87245 & .96811 & 0.000 \\
\hline & Uncooled gel post & 16 & 96.0625 & 2.54214 & .63554 & 0.000 \\
\hline \multirow[b]{2}{*}{ Po2 } & Cooled gel post & 16 & 95.3125 & 2.12034 & .53008 & 0.037 \\
\hline & Uncooled gel post & 16 & 97.0000 & 2.25093 & .56273 & 0.037 \\
\hline
\end{tabular}

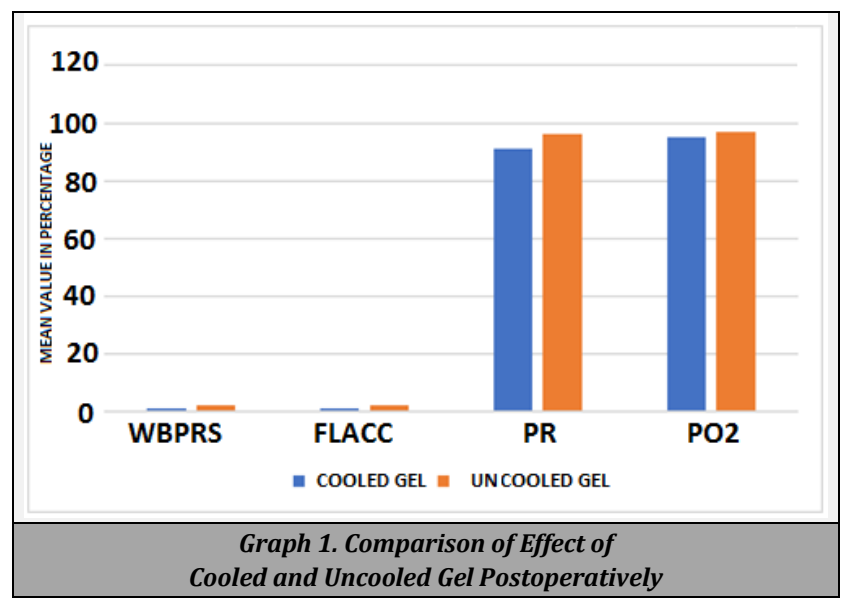

\section{DISCUSSION}

Reduction in the pain perception is of paramount importance in paediatric dentistry to achieve optimum comfort for patient. The effect of application of topical gel with and without cooling were evaluated in this study. Cryoanaesthesia is a unique technique which is used to reduce the sensation of pain and works by the principle of blocking local neural transmission of painful stimulus by cooling a localized area. The reduced temperature stimulates myelinated 'A' fibers and activates pain pathways of inhibition, and thus succeeds in raising the pain threshold of an individual. ${ }^{6}$ Moreover cooling of the tissues locally is also believed toslow or eliminate pain signal transmission and also cause retardation in neuromuscular transmission. In addition reduction of muscle tone due to a decrease in the activity of the spindles of muscle tissue occurs. ${ }^{8}$

An age group of 8 - 10 years was considered as they were expected to provide a reliable and valid report of their pain. Dental extractions which only required infiltration anaesthesia were chosen for the present study as diffusion ability of the anaesthetic agent decreases as the distance increases. Hence, anterior maxillary region was ideal due to its porous nature for studies on anaesthetic efficacy. ${ }^{9}$

The topical gel was applied for an optimum time of 1 minute for its adequate action. The objective and subjective parameters were considered in this study. According to literature, suggested application time was 2 - 5 minutes. It was reported that five minutes could be well tolerated by adult patients, but in young patients it may further lead to lack of cooperation. ${ }^{6}$ Therefore, in the present study cooled gel was applied on the buccal mucosa just for $1 \mathrm{~min}$. In the present study, topical anaesthetic gel was cooled to $4^{\circ} \mathrm{C}$ as overcooling could make the patient uncomfortable. Cooling was done to make the procedure more physiologically effective. The sensation caused by cooling was more familiar to the patients and hence less likely caused discomfort, anxiety or fear, especially in young individuals. Wong Bakers faces pain rating scale is represented by 6 faces depicting increase in degree of pain from left to right. Each face indicates a score of 0 - 10 on the scale. Patients were taught the meaning of each face as 0 - does not hurt at all, 2 - hurts just a little bit, 4 - hurts a little more, 6 - hurts even more, 8 hurts a whole lot, 10 - hurts as much as you can imagine. ${ }^{10}$

The pulse rate and oxygen saturation were assessed as objective and more reliable indicators of anxiety and fear. An increase in heart rate was the most common physiologic indicator for anxiety and fear. ${ }^{11}$

Harbert ${ }^{5}$ demonstrated that pre-cooling the soft tissues prior to palatal injection was an efficient method to relieve the pain caused during needle insertion. Whereas other reports by Duncan ${ }^{12}$ et al. ${ }^{9}$ stated that application of a cotton pellet saturated with dichlorodifluoromethane spray for 5 seconds prior to administrating palatal injections revealed less discomfort during needle penetration and similar results. Other studies by Kosaraju et al. ${ }^{13}$ successfully compared application of a refrigerant spray for 5 seconds to application of a topical gel before local anaesthetic injection in the posterior palatal site for 2 minutes using a 30 - gauge needle. Their results indicated that the application of refrigerant agent prior to anaesthetic injection was more effective than the topical gel application. Comparison of ice and local anaesthetic gel before injection was done by Mohiuddin et al. ${ }^{14}$ for procedures involving the extraction of primary maxillary anterior teeth and he concluded that the use of ice was very effective. A study done by Lathwal et al. ${ }^{7}$ revealed that ice was superior, demonstarting higher efficacy compared to benzocaine and refrigerant sprays during intraoral injections. Wiswall et al. ${ }^{15}$ surveyed the response to pain caused in three different site preparations [pressure, pressure + topical anaesthetic (20\% benzocaine), and pressure + precooling] prior to the greater palatine nerve block. Their reports suggested that there was no significant VAS differences between the test groups and concluded that all of them were almost equally effective. The findings of Gadheri 16 et al. also suggest that cooling the injection site prior to infiltration anaesthesia in the buccal mucosa for a minimum of 1 minute, reduced the pain perception in paediatric patients. The results of the present study showed cooling the anaesthetic gel has statistically significant reduction in pain perception during injection procedure and may also be helpful in patient management during dental procedures. 


\section{CONCLUSIONS}

Application of cooled topical gel has shown significant decrease in the perception of pain during infiltration in children during routine dental procedures. Use of such a technique is advised to make the method of local anaesthetic administration more undemanding, reliable and economical and can be used as an adjunctive procedure for the same.

However, the present study design was not able to support a double - blind pattern. The principle of interactions of anaesthetic gel and cold as two separate mechanisms for reducing injection pain could not be considered as well.

Data sharing statement provided by the authors is available with the full text of this article at jemds.com.

Financial or other competing interests: None.

Disclosure forms provided by the authors are available with the full text of this article at jemds.com.

\section{REFERENCES}

[1] Chaudhry K, Shishodia M, Singh C, et al. Comparative evaluation of pain perception by vibrating needle (Vibraject ${ }^{\mathrm{TM}}$ ) and conventional syringe anaesthesia during various dental procedures in paediatric patients: a short study. Int Dent Med J Adv Res 2015;1:1-5.

[2] Raslan N, Masri R. A randomized clinical trial to compare pain levels during three types of oral anaesthetic injections and the effect of Dentalvibe $\AA$ on injection pain in children. Int J Paediatr Dent 2018;28(1):102-10.

[3] Meechan JG. Intra-oral topical anaesthetics: a review. J Dent 2000;28(1):3-14.

[4] Bhalla J, Meechan JG, Lawrence HP, et al. Effect of time on clinical efficacy of topical anaesthesia. Anesth Prog 2009;56(2):36-41.

[5] Harbert H. Topical ice: a precursor to palatal injections. J Endod 1989;15(1):27-8.

[6] Aminabadi NA, Farahani RMZ. The effect of pre-cooling the injection site on paediatric pain perception during the administration of local anaesthesia. J Contemp Dent Pract 2009;10(3):43-50.

[7] Lathwal G, Pandit IK, Gugnani N, et al. Efficacy of different precooling agents and topical anaesthetics on the pain perception during intraoral injection: a comparative clinical study. Int J Clin Paediatr Dent 2015;8(2):119-22.

[8] Ottoson D. The effects of temperature on the isolated muscle spindle. J Physiol 1965;180(3):636-48.

[9] Isik K, Kalayci A, Durmus E. Comparison of depth of anaesthesia in different parts of maxilla when only buccal anaesthesia was done for maxillary teeth extraction. Int J Dent 2011;2011:575874.

[10] Khatri A, Kalra N. A comparison of two pain scales in the assessment of dental pain in East Delhi children. ISRN Dent 2012;2012:247351.

[11] Kadhim NA. Evaluation of anxiety, fear and physiological responses among children with and without presence of their parents in dental clinic during dental treatment. International Journal of Science and Research 2018;7(1):698-704.

[12] Duncan JD, Reeves GW, Fitchie JG. Technique to diminish discomfort from the palatal injection. J Prosthet Dent 1992;67(6):901-2.

[13] Kosaraju A, Vandewalle KS. A comparison of a refrigerant and a topical anaesthetic gel as pre injection anaesthetics: a clinical evaluation. J Am Dent Assoc 2009;140(1):68-72.

[14] Mohiuddin I, Setty JV, Srinivasan I, et al. Topical application of local anaesthetic gel vs ice in paediatric patients for infiltration anaesthesia. J Evol Med Dent Sci 2015;4(74):12934-40.

[15] Wiswall AT, Bowles WR, Lunos S, et al. Palatal anaesthesia: comparison of four techniques for decreasing injection discomfort. Northwest Dent 2014;93(4):25-9.

[16] Ghaderi F, Banakar S, Rostami S. Effect of pre-cooling injection site on pain perception in paediatric dentistry: a randomized clinical trial. Dent Res J (Isfahan) 2013;10(6):790-4. 